\title{
A Novel Route Guidance Algorithm with Maximum Coverage and Minimum Handover for Vehicular Networks
}

\author{
Mohamed Chedly Ghedira, Walid Ben-Ameur and Hossam Afifi \\ Wireless Networks and Multimedia Services Department of TELECOM SudParis, Evry, France \\ \{Mohamed-Chedly.Ghedira | Walid.Benameur |Hossam.Afifi\}@int-edu.eu
}

\begin{abstract}
In this paper, we propose a novel route guidance algorithm that provides better wireless network availability. Using a GPS device, geographical information is considered to select a short geographical path while ensuring the maximum covered distance and the minimum handovers number. An efficient algorithm is derived to tackle the problem in polynomial time. Our solution is optimal and well suited to all possible scenarios including low and high hotspots density. By comparing our algorithm with some common practices, we minimize significantly the handovers number and decrease the wireless uncovered distance, with a negligible overhead in the traversed distance.
\end{abstract}

\section{Introduction}

Route guidance is a way to direct vehicle drivers from the current location to a given destination. It has become into wide use recently. Authors in [1] prove that automated vehicles could improve the network efficiency in comparison with manually driven vehicles. To ensure a good direction of automated vehicles, a suitable route guidance system is needed. In many cases, these systems take profit from information given by a GPS [2] (Global Positioning System). Furthermore, the placed hotspots along routes could be exploited by route guidance systems. In general, these hotspots rely on the IEEE 802.11 technology which has a limited communication range conducting mobile users to perform frequent handovers.

Moving from a place to another may require a frequent change of cells, clients have to perform transparent handovers in order to ensure a seamless mobility with a minimal packet loss. Numerous researches were done to determine a route guidance algorithm responding to user criteria, leading to a very large variety of algorithms. However, while proposed route guidance systems have many differences in term of design, most of them aim to minimize the travel distance or the travel time of a vehicle moving from an origin to a destination, regardless of the hotspots locations. In other words, they attempt to avoid traffic congestion and to determine the quickest route on the roadway network, without considering the coverage and the number of handovers.

In [3], authors evaluate 15 shortest path algorithms in real road networks of 10 states of the United Sates. They identify a set of recommendations regarding algorithm selection based on the computational performance. Their findings show that Pallotino's Graph Growth Algorithm has the best performance for solving the one-to-all shortest path problem. For the shortest paths from a source node to a subset of the nodes (one-to-some), authors suggest to consider one of the Dijkstra's implementations: the Dijkstra approximate buckets implementation (DIKBA) or the Dijkstra double buckets (DIKBD) implementation. They also show that certain implementations should be avoided when solving shortest paths on real networks.

In [4], authors use neurodynamic programming to design a dynamic route guidance algorithm. Their system uses probe vehicles (GPS-equipped taxis) that collect data. The travel time of the probe vehicle in the road links is then determined. This method permits to avoid routes with important traffic flow.

In [5], authors introduce the use of a central server. Drivers send periodically information to the server about their future traversed routes. The server accumulates all the information, calculates the prospective traffic, and then sends it to the drivers. After receiving it, each driver reconsiders and changes the route. Simulations show that the travel time of both users using this mechanism and the other drivers decreased and that the travel time of drivers using the proposed system is shorter than those of drivers using other mechanisms.

Authors in [6] propose a decentralized system. Each driver has a default path to reach a certain destination. The information received from other vehicles can be used to change this path based on the current status of the network. 
A route guidance system aims to select the best route between a given source to a given destination. This selection has to be based on the user satisfaction level. If the user considers only the traversed distance, he will be satisfied with the shortest geographical route. However, the shortest route is not the unique criteria to satisfy users. In many cases, other criteria come into play, such as vehicular traffic or the maximum covered distance ensuring internet access. Even if the selected route is a little bit longer than the shortest one, the user can be more satisfied with this result. Thought the large number of researches in route guidance systems, we were not able to find in literature any work about how to compute a short geographical path while ensuring a large covered distance and a low number of handovers.

Most existing routing algorithms in vehicular networks focus on avoiding traffic congestion by finding a shortest path in terms of distance or travel time and do not take into account the minimization of the number of handovers from a cell to another. This often conducts to an important number of handovers which will increase latency and packet loss.

In this paper, we exploit geographical information given by a GPS device to derive an algorithm ensuring a low number of handovers while moving from a position to another based on the hotspot map. In addition, the algorithm minimizes both the total travel distance and the uncovered distance.

In our approach, the mobile uses its location information and the hotspot map to build an oriented graph representing different portions of routes and cells. Every node of the graph represents a portion of a route covered by a hotspot.

The edges of the graph represent possible routes between nodes with a weight function taking into account the handover cost and the cost of both covered and uncovered distance, instead of the classical weight of the geographical path distance. Applying a classical shortest path algorithm to this graph enables the mobile to decide proactively to which network it has to switch and which path it has to follow, in order to increase the covered distance, decrease the number of handovers while the traversed distance is kept under control.

Moreover, our algorithm can be used by an operator to determine the best locations to place its hotspots.

The rest of this paper is organized as follows. In Section II, we describe our algorithm. Section III presents some computational experiments to evaluate the performance of our proposal and we give some details about the complexity of the algorithm. Finally, this is followed by some concluding remarks and further research directions.

\section{Proposed algorithm}

In this section, we describe our proposition.

\subsection{The algorithm steps}

It is divided into four main steps:

- First, we determine intersections between segments (corresponding to routes) and circles (representing hotspot coverage). This leads us to divide the existing segments into many other sub segments limited by intersections points. An example is shown in Figure 1 where segments are enumerated from $\mathrm{S} 1$ to $\mathrm{S} 12$.

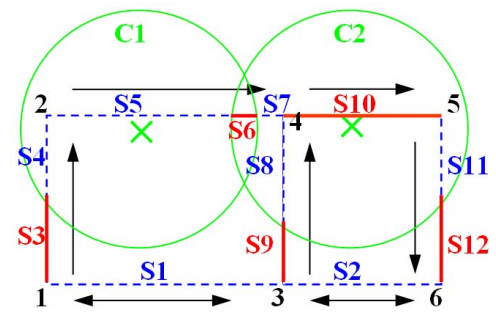

Figure 1. An example of a roads map

- Then, we build an oriented graph $\mathrm{G}(\mathrm{V}, \mathrm{E})$, where $\mathrm{V}$ is a set of nodes and each node represents a couple of a segment and a cell. Since a segment can be covered by a certain number $\mathrm{N}$ of cells, there will be $\mathrm{N}$ nodes corresponding to this segment. For example, segment $\mathrm{S} 6$ is covered by 2 cells: $\mathrm{C} 1$ and $\mathrm{C} 2$. This will be translated by two nodes in the graph: $(\mathrm{S} 6, \mathrm{C} 1)$ and $(\mathrm{S} 6, \mathrm{C} 2)$. E is a set of edges, where each edge represents a one-way road. Twoways roads are represented by two edges, one in each direction (see, e.g., S1 and S2). The corresponding graph of Figure 1 is shown in Figure 2. Nodes are represented by couples (Segment, Cell). We mark the cell number as zero if the segment is not covered by any cell.

- Once the two first steps are done, we add two nodes to the graph G. The first node represents the source node which is the current location. The second one corresponds to the destination. We add necessary edges connecting these new nodes to potential future nodes. For this example, we suppose that a vehicle is going from vertex V1 (the intersection of S1 and S3) to vertex V6 (intersection of S2 and $\mathrm{S} 12)$. The resulting graph is shown in Figure 3.

- The final step consists in computing the best path from the current location to the destination using a classical shortest path algorithm such as for example the Dijkstra algorithm[7] and considering a weight depending on the geographical distance, uncovered distance and number of handovers. 


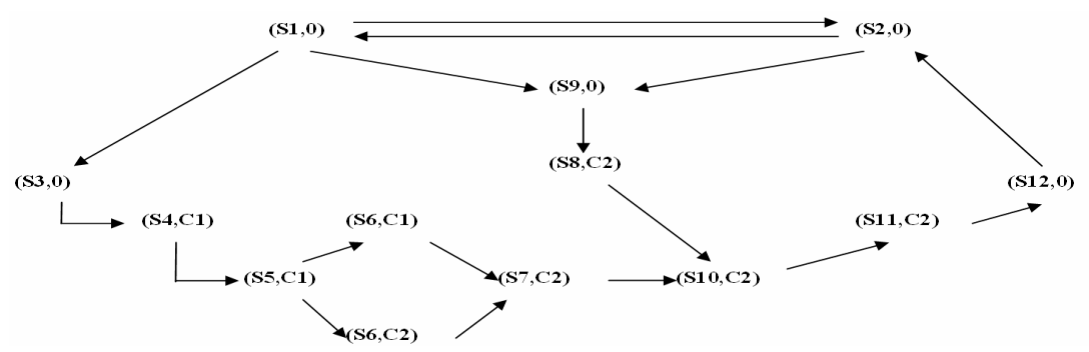

Figure 2. The corresponding graph of the considred roads map

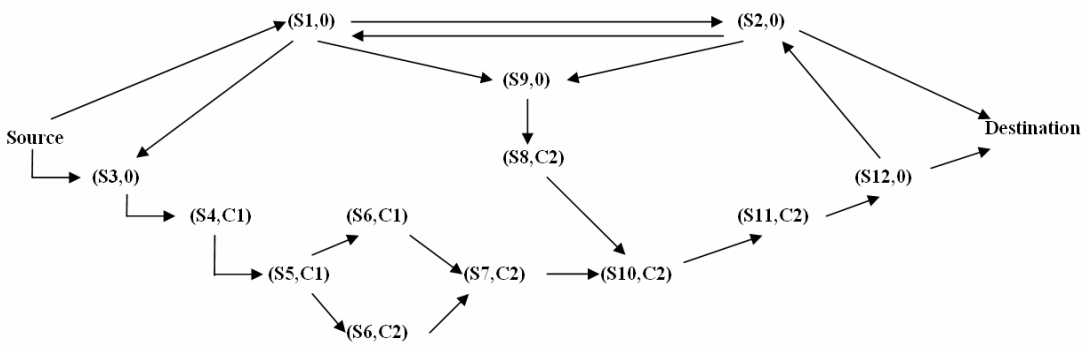

Figure 3. The corresponding graph after adding the source and the destination

It is clear that any path linking the source to the destination in the graph of Figure 3 represents both a geographical path and a certain choice of the hotspots.

Our algorithm can be implemented in a GPS device to enhance the way of calculating the path between the current position and a given destination. We assume that the town map is known. This is a natural assumption since that all GPS devices need the town map to calculate paths. We also assume that the hotspots map is known. This information is already used by some tools such as Mappy[8].

In addition to the fact that our algorithm improves the quality of service since it decreases the number of handovers and increases the covered distance, it can also be used to decrease the handover latency. This can be done by performing a fast proactive handover, since our solution determines proactively the next hotspot the user will switch to.

\subsection{Weights}

Weights (or costs) associated to the edges follow this formula:

Where:

$$
(\alpha * \mathrm{Dc})+(\beta * \mathrm{Ho})+(\lambda * \mathrm{Dnc})
$$

- Dc is the covered distance. So Dc is equal to the geographical distance if the considered segment is covered. Otherwise, we will have $\mathrm{Dc}=0$.

- Dnc is the uncovered distance. So Dnc is equal to the geographical distance if the considered segment is not covered by any hotspot. Otherwise, we will have Dnc $=0$.
- Ho represents the cost of a handover. We take $\mathrm{Ho}=1$ if we are making a handover (still covered but changing the current cell). We also have $\mathrm{Ho}=1$ if we are moving from a covered segment to an uncovered one. This is to prioritize the choice of the continuous coverage (Figure 4.a) on the discontinuous coverage (Figure 4.b), even if the total covered distance is the same.

Ho $=0$, otherwise i.e. if we are moving inside the coverage of the same hotspot. We also take Ho $=0$ either if we are moving from a uncovered segment to a covered one, or if we are moving from a uncovered segment to a uncovered one.

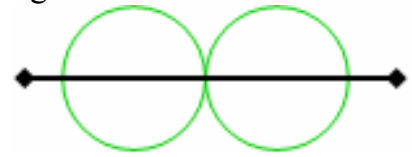

Figure 4. A route having a continuous coverage

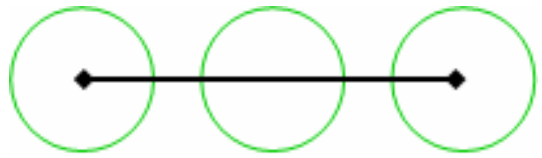

Figure 5. A route having a discontinuous coverage

$\alpha, \beta$ and $\lambda$ are the weighting coefficients. Note that $\lambda>\alpha$ to prioritize the choice of a covered distance on an uncovered one.

The choice of the weighting coefficients is crucial. It strongly depends on the client wishes in terms of priorities (maximum covered distance, shortest geographical path, etc.). In the example above, there are three paths to reach the destination. Following the shortest path, the user will take the itinerary 
$\mathrm{V} 1 \rightarrow \mathrm{V} 3 \rightarrow \mathrm{V} 6$ but the path will be not covered at all. Another existed path: $\mathrm{V} 1 \rightarrow \mathrm{V} 3 \rightarrow \mathrm{V} 4 \rightarrow \mathrm{V} 5 \rightarrow \mathrm{V} 6$ with better coverage. The path presenting the best coverage is $\mathrm{V} 1 \rightarrow \mathrm{V} 2 \rightarrow \mathrm{V} 4 \rightarrow \mathrm{V} 5 \rightarrow \mathrm{V} 6$, but the traversed distance will be longer. This is why a performance evaluation is needed to measure the impact of changing the weighting coefficients on the total length of the path, the number of handovers and the uncovered distance.

\section{Performance evaluation}

In this section, we present some analysis and simulation results to evaluate the performance of our algorithm. For that, we have considered a typical roadmap constituted of a grid with 50 crossings. The distance between two adjacent crossings is 200 meters. Hotspots are randomly distributed. Each hotspot is assumed to cover a disk whose radius is 200 meters.

We consider two scenarios:

- In the first scenario, we run the algorithm to determine which path to follow. This is useful when, for example, the GPS device is used in a car, or more specifically a taxi.

- In the second one, we assume that the geographical path is fixed. This is the case for example if the user is in a metro, bus, train, etc.

For each scenario, we will consider three types of hotspot densities: low (less than 25 hotspots), moderate and high (all routes are covered on average by more than one hotspot). For each one of these densities, the obtained values are the average of 15 hotspots distributions, with 20 different sources and destinations for each distribution. While the first scenario is useful to evaluate the general performance of our algorithm, the second scenario will only focus on the minimization of the number of handovers through a given path.

In the first scenario, the path obtained by our algorithm is compared with the shortest path (in terms of geographical distance). Many experiments are carried out by taking different values of the weighting coefficients $\alpha, \beta$ and $\lambda$. Since multiplying all coefficients by a positive number does not change the path, we take $\alpha=1$. Then, $\beta$ varies between 0 and 10 , and $\lambda$ varies between 1 and $10(\alpha<\lambda$ to ensure a priority to covered routes).

In Figures 6, 7 and 8, the y-axis values are calculated as follows:

$$
100 *\left[\operatorname{Dnc}(\mathrm{SP}) / \mathrm{D}(\mathrm{SP})-\operatorname{Dnc}\left(\mathrm{P}_{\lambda, \beta}\right) / \mathrm{D}\left(\mathrm{P}_{\lambda, \beta}\right)\right]
$$

Where SP is the classical shortest path, $\mathrm{P}_{\lambda, \beta}$ is the path obtained by our algorithm, Dnc is the length of the uncovered portion of the path (in both cases) and D is the total length of the path $(\mathrm{D}=\mathrm{Dc}+\mathrm{Dnc})$. Notice that the shortest path $\mathrm{SP}$ is nothing else than $\mathrm{P}_{1,0}$.
Formula (2) clearly evaluates the improvement in terms of uncovered distance when our algorithm is compared to the classical shortest path algorithm.

We notice the following:

- For low and moderate density environments, the behaviours of curves are similar. All curves are decreasing when $\beta$ grows up. This is quite expectable since the increase of $\beta$ discourages the algorithm to perform frequent handovers and so, can avoid some covered routes to maximize the objective function.

- When $\lambda$ is small $(\lambda=1,2)$, the uncovered distance is not well represented in the objective function. This leads to bad performance in terms of uncovered distance when $\beta$ increases.

- Independently of $\beta$ value, our algorithm performs better when increasing $\lambda$. It allows about $20 \%$ decrease in terms of uncovered distance when compared to the shortest path algorithm.

- For a very dense environment, all values are the same. This is explained by the fact that, in such an environment, all paths are covered.

In Figures 9, 10 and 11, the y-axis values are given by:

$$
100 *\left[\mathrm{D}\left(\mathrm{P}_{\lambda, \beta}\right) / \mathrm{D}(\mathrm{SP})\right]
$$

Formula (3) represents the increase in terms of the total traversed distance.

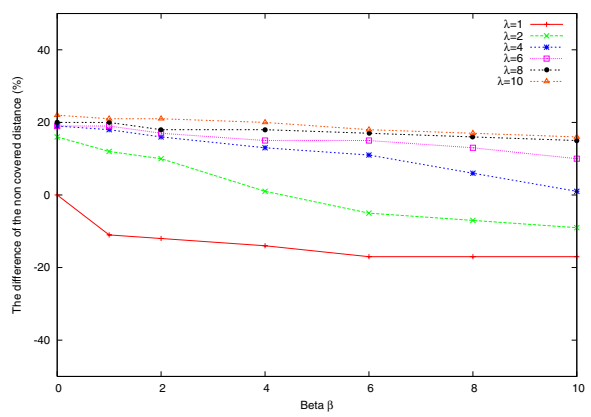

Figure 6. Improvement of uncovered distance in a low density environment

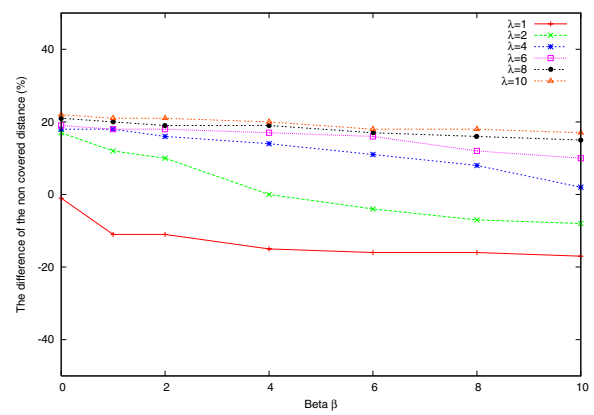

Figure 7. Improvement of uncovered distance in a moderate density environment 


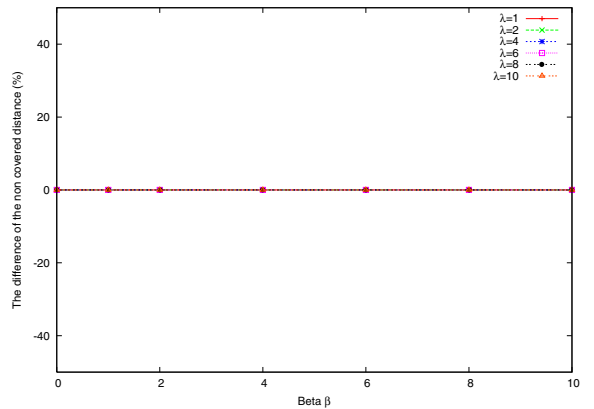

Figure 8. Improvement of uncovered distance in a high density environment

We notice the following:

- For low and moderate density environments, one can see that the traversed distance decreases when $\beta$ increases and $\lambda>2$. In fact, when $\lambda$ is large, the weight of the uncovered distance is important leading to longer paths. When $\beta$ increases, this phenomenon is attenuated since that the minimization of the number of handovers generally lead to shorter paths.

- When $\lambda<=2$ and $\beta$ is small, the traversed distance is close to the length of the shortest path. This is mainly due to the fact that the number of handovers and the uncovered distance are not well represented in the objective function. However, when $\beta$ increases the traversed distance increases due to the introduction of the number of handovers in the cost function.

- Whatever the chosen values of $\beta$ and $\lambda$ are, the added traversed distance does never exceed in the worst case $16,5 \%$ for a low density environment and $12 \%$ for a moderate density environment. This may be considered as a positive result: we can maintain coverage and minimize the number of handovers only with a small additional traversed distance.

- For a high density environment, all paths are covered. The path obtained by the algorithm is a shortest path.

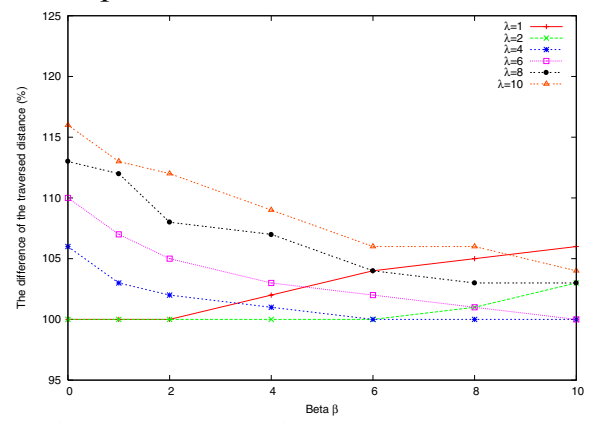

Figure 9. Variations of the total traversed distance in a low density environment

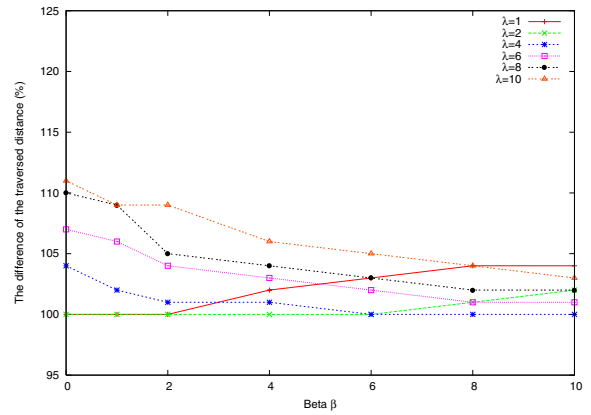

Figure 10. Variations of the traversed distance in a moderate density environment

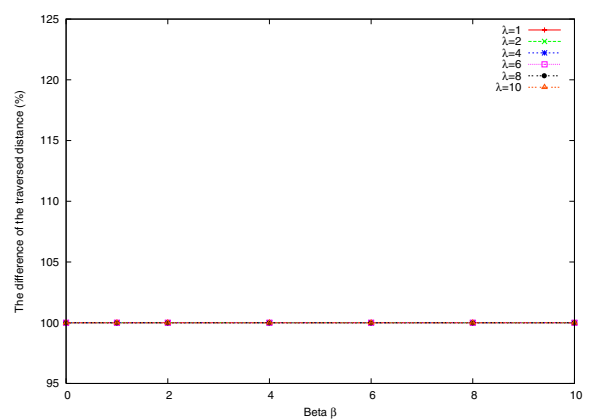

Figure 11. Variations of the total traversed distance in a high density environment

Some details about the theoretical complexity of the algorithm are given below.

As explained in section III, there are 4 steps in the algorithm. The two first steps conducting to the construction of the graph are done once, and the result is preloaded in the used machine (such as the GPS device). This is why, we don't take into account its complexity. Remain the two other steps: adding the source and destination nodes and edges, and running the shortest path algorithm on the resulting graph.

Let us denote:

- R: the number of routes.

- H: the average number of hotspots covering a route.

Notice that a route is a segment between two consecutive crossings. $\mathrm{H}$ is generally a small number.

The total number of nodes in the oriented graph will be about $\mathrm{O}\left(\mathrm{R}^{*} \mathrm{H}\right)$. It is well known that the time complexity of Dijkstra's algorithm for a graph G (V, $E)$, is about $O(|E|+|V| \log |V|)[9]$. So for our graph, the complexity will be less than $\mathrm{O}\left(\left(\mathrm{R}^{*} \mathrm{H}\right)^{2}\right)$.

For sake of simplicity, in this paper we have focused on static parameters, but our algorithm can also be easily extended to a dynamic route guidance algorithm by adding new terms in the cost function depending on the current conditions (the current position of the user, signal strength, or the number of connected users to each hotspot, etc). It is important to notice that this does not increase the complexity of the algorithm. We still have to compute a shortest path. 
In the second scenario, we compare our algorithm with two other approaches that we call ABS (Always Best Signal) and ECBS (End Coverage Best Signal). We have chosen these two algorithms for comparison because most of existing approaches for selecting hotspots are based on the signal strength. The ABS approach consists of, at every moment, selecting the hotspot having the best signal. The ECBS approach does not change its current hotspot unless it reaches the end of its cover, and so select the hotspot with the best signal.

We have also considered three environments with different hotspots densities. Table 1 presents a comparison of the total number of handovers (changing the current hotspot) for the three algorithms in the three considered environments. For each experiment, a set of values of $\beta$ and $\lambda$ is chosen and used to compute a path $\mathrm{P}_{\lambda, \beta}$. Then algorithms ABS and ECBS are considered to compute the number of handovers when the used path is exactly $\mathrm{P}_{\lambda, \beta}$. results are compared with the number of handovers obtained by our algorithm.

The first column of Table 1 corresponds to the ratio of the number of handovers obtained when our algorithm is used (denoted by Algo) to the same number when ABS is used. One can notice that this ratio decreases when the density increases. This is explained by the fact that in a very dense environment, many hotspots have common coverage, so there is a need for an intelligent algorithm to make the choice between hotspots in order to minimize handovers thereafter. Whereas the ABS algorithm chooses the best hotspot even if the user will move out of its range in few seconds.

TABLE I. Total number of handovers

\begin{tabular}{|l|c|c|c|}
\cline { 2 - 4 } \multicolumn{1}{c|}{} & $\begin{array}{c}\mathbf{1 0 0}^{*} \\
\text { (Algo/ABS) }\end{array}$ & $\begin{array}{c}\mathbf{1 0 0}^{*} \\
\text { (Algo/ECBS } \\
\text { ) }\end{array}$ & $\begin{array}{c}\mathbf{1 0 0}^{*} \\
\text { (ECBS/ ABS) }\end{array}$ \\
\hline Low density & 50.48 & 60.52 & 83.41 \\
\hline Moderate density & 46.73 & 65.59 & 71.25 \\
\hline High density & 33.06 & 59.85 & 55.24 \\
\hline
\end{tabular}

The second column is comparing our solution and ECBS. We notice that whatever the environment is, our algorithm is always better. ECBS is of course better than ABS: it does not change its current hotspot unless it reaches the end of its coverage. This can lead to a ping pong effect: When a user is moving inside the coverage of many hotspots, he is changing his current hotspot back and forth among them since the signal strength favours different hotspots at different points. This is verified by comparing ABS and ECBS in the third column.

\section{Conclusion}

In this paper, we have described a new guidance algorithm that uses geographical information to route vehicles towards a destination. It studies how to minimize the combination of path distance and number of handovers while maximizing coverage and connectivity. We tested the behaviour of our algorithm in environments with different hotspot densities and we compared it with previously proposed solutions.

The algorithm is simple, divided into four steps (as described in Section III). Its performance depends on two weighting parameters $\beta$ and $\lambda$, which can be chosen in according to the user priorities.

Different paths ( 2 to 5 ) can be computed using different values of $\beta$ and $\lambda$. These paths can be presented to the user with some information about the total length of the path, the uncovered distance and the number of handovers. Then the user will choose the best path for him. It is also important to notice that the complexity of the algorithm is quite reduced even if it is applied 5 times with different values of $\beta$ and $\lambda$.

Future work will focus on increasing the coverage by creating an AdHoc network between vehicles which will forward packets to other uncovered users. We will concentrate in defining a new proactive handover procedure using a mesh network between hotspots.

\section{References}

[1] S. Kim and J. Lee, "A study on design of dynamic route guidance system using forecasted travel time based on GPS data and modified shortest path algorithm" ITSC 1999, pp. 44-48.

[2] E. Murakami, and D. Wagner, "Can using global positioning system (GPS) improve trip reporting?" Transportation Research Part C: Emerging Technologies, vol. 7, pp. 149-165, 1999.

[3] F.B.Zhan, and C.E.Noon, "Shortest path algorithms: an evaluation using real road networks," Transportation Science, vol. 32, pp. 65-73, 1998.

[4] W. Zhang, Z. Zhang and J. Xu, "Dynamic route guidance using neurodynamic programming" ISDA 2006. Volume: 2 Oct. 2006 pp. 1177-1181.

[5] T. Yamashita, K. Izumi, and K. Kurumatani, "Car navigation with route information sharing for improvement of traffic efficiency," ITSC 2004, pp. 465- 470.

[6] J. Farver and I. Chabini, "A vehicle-centric logic for decentralized and hybrid route guidance," ITSC 2003, vol.2 pp. $1627-1632$.

[7] E.W. Dijkstra, "A note on two problems in connexion with graphs" Numerische Mathematik, vol. 1, p. 269, 1959

[8] Mappy, URL: http://www.mappy.com/, June 28, 2007.

[9] B. Korte and J. Vygen, "Combinatorial optimization: theory and algorithms." No. 21 in Algorithmics and Combinatorics, New York: Springer Verlag, 3 ed., 2005. 